\section{NCOA4-mediated ferritinophagy in macrophages is crucial to sustain erythropoiesis in mice}

\author{
Antonella Nai, ${ }^{1,2}$ Maria Rosa Lidonnici, ${ }^{3}$ Giorgia Federico, ${ }^{4}$ Mariateresa \\ Pettinato,,$^{1,2}$ Violante Olivari, ${ }^{1}$ Federica Carrillo, ${ }^{4,5}$ Simonetta Geninatti Crich, ${ }^{6}$ \\ Giuliana Ferrari, ${ }^{2,3}$ Clara Camaschella, ${ }^{1}$ Laura Silvestri ${ }^{1,2 \#}$ and Francesca \\ Carlomagno ${ }^{4,5 \#}$
}

${ }^{1}$ Division of Genetics and Cell Biology, San Raffaele Scientific Institute, Milan; ${ }^{2}$ VitaSalute San Raffaele University, Milan; ${ }^{3}$ SR-TIGET, San Raffaele Scientific Institute, Milan; ${ }^{4}$ Department of Molecular Medicine and Medicine Biotechnology (DMMBM), University of Naples Federico II, Naples; ${ }^{5}$ Institute of Endocrinology and Experimental Oncology (IEOS), CNR, Naples and ${ }^{6}$ Department of Molecular Biotechnology and Health Sciences, University of Turin, Turin, Italy

${ }^{\#} \mathrm{LS}$ and FrC contributed equally as co-senior authors.
$\mathrm{N}$ uclear receptor coactivator 4 (NCOA4) promotes ferritin degradation and Ncoa4-ko mice in a C57BL/6 background show microcytosis and mild anemia, aggravated by iron deficiency. To understand tissue-specific contributions of NCOA4-mediated ferritinophagy we explored the effect of Ncoa4 genetic ablation in the iron-rich Sv129/J strain. Increased body iron content protects these mice from anemia and, in basal conditions, Sv129/J Ncoa4-ko mice show only microcytosis; nevertheless, when fed a low-iron diet they develop a more severe anemia compared to that of wild-type animals. Reciprocal bone marrow (BM) transplantation from wild-type donors into Ncoa4-ko and from Ncoa4-ko into wild-type mice revealed that microcytosis and susceptibility to iron deficiency anemia depend on BM-derived cells. Reconstitution of erythropoiesis with normalization of red blood count and hemoglobin concentration occurred at the same rate in transplanted animals independently of the genotype. Importantly, NCOA4 loss did not affect terminal erythropoiesis in iron deficiency, both in total and specific BM Ncoa4-ko animals compared to controls. On the contrary, upon a low iron diet, spleen from wild-type animals with Ncoa4-ko BM displayed marked iron retention compared to (wild-type BM) controls, indicating defective macrophage iron release in the former. Thus, erythropoietin administration failed to mobilize iron from stores in Ncoa4-ko animals. Furthermore, Ncoa4 inactivation in thalassemic mice did not worsen the hematologic phenotype. Overall our data reveal a major role for NCOA4-mediated ferritinophagy in macrophages to favor iron release for erythropoiesis, especially in iron deficiency.

\section{Introduction}

Nuclear receptor coactivator 4 (NCOA4), originally identified as an androgen receptor interactor, ${ }^{1}$ is a novel player in iron metabolism contributing to the regulation of cell and systemic iron homeostasis. NCOA4 acts as a cargo receptor that promotes "ferritinophagy", the selective autophagy-mediated degradation of the iron storage protein ferritin., ${ }^{2,3}$ This process is induced in conditions of iron deficiency to facilitate iron recovery from intracellular stores. ${ }^{4,5}$

As expected, inactivation of Ncoa4 increases cell ferritin aggregates ${ }^{2-4}$ and C57BL/6 Ncoa4-knockout ( $k 0$ ) mice show ferritin and iron accumulation in several organs, in particular splenic macrophages. ${ }^{3,6}$ In addition, they display a mild microcytic anemia and develop a more severe anemia than wild-type (wt) mice when fed
Haematologica 2021

Volume 106(3):795-805

\section{Correspondence:}

FRANCESCA CARLOMAGNO francesca.carlomagno@unina.it

LAURA SILVESTRI

silvestri.laura@hsr.it

Received: October 30, 2019.

Accepted: February 25, 2020.

Pre-published: February 27, 2020.

https://doi.org/10.3324/haematol.2019.241232

(C)2021 Ferrata Storti Foundation

Material published in Haematologica is covered by copyright. All rights are reserved to the Ferrata Storti Foundation. Use of published material is allowed under the following terms and conditions:

https://creativecommons.org/licenses/by-nc/4.0/legalcode. Copies of published material are allowed for personal or internal use. Sharing published material for non-commercial purposes is subject to the following conditions:

https://creativecommons.org/licenses/by-nc/4.0/legalcode, sect. 3. Reproducing and sharing published material for commercial purposes is not allowed without permission in writing from the publisher. 
an iron-deficient diet. ${ }^{6}$ Whether this phenotype is due to an intrinsic defect of erythroid cells or to retention of stored iron because of impaired ferritin degradation due to Ncoa4 inactivation is a matter of investigation.

Some evidence argues in favor of an intrinsic erythroid function for NCOA4. First, NCOA4 is expressed at high levels in maturing orthochromatic erythroblasts; ${ }^{7}$ second, in vitro ${ }^{4,8}$ and ex vivo data suggest that NCOA4 is required for the differentiation and hemoglobinizationof erythroid cells, modulating iron incorporation into heme. An erythropoietic role for NCOA4 was also suggested in vivo in zebrafish embryos treated with morpholinos to Ncoa4. ${ }^{4} \mathrm{~A}$ moderate-to-severe anemia was observed in Ncoa4-ko mice at birth, which was mostly rescued in adult animals. ${ }^{9,10}$ Although all these findings suggest a role for NCOA4 in erythropoiesis, formal proof that anemia of adult Ncoa4-ko mice is due to loss of protein activity in erythroid cells is still lacking. Recent data ${ }^{10}$ point toward both an autonomous and non-autonomous effect of NCOA4 in erythropoiesis. A condional tamoxifeninduced total Ncoa4-ko and a tissue-specific one (through the expression of CRE-recombinase under the control of the erythropoietin receptor promoter in Ncoa4-floxed transgenic animals) were generated by Santana Codina et al. However, both tamoxifen-induced toxic effects on red blood cells ${ }^{11}$ and non-erythroid-restricted expression of erythropoietin receptor ${ }^{12}$ still leave the question open.

In order to clarify the in vivo function of NCOA4, its role in erythropoiesis and to identify the cell type mostly affected by Ncoa4 deficiency in vivo we used different approaches. First we generated Ncoa4-ko mice on the ironrich Sv129/J strain and analyzed the animals in basal conditions and after different challenges. We then performed reciprocal transplantation of bone marrow from wt and Ncoa4-ko mice into Ncoa4-ko and wt mice. Finally, we crossed Ncoa4-ko with $\mathrm{Hbb}^{\text {thlit+ }}$ mice, a model of transfusion-independent $\beta$-thalassemia. We proved that reduced iron release by macrophages is the principal driver of anemia in Ncoa4-ko animals and excluded a relevant role for NCOA4 in erythroid cells in vivo.

\section{Methods}

\section{Mouse models and bone marrow transplantation}

Ncoa4-ko mice on a Sv129/J background were generated as described by Bellelli et al. ${ }^{6}$ and in the Online Supplementary Materials. Wild-type littermates were used as controls in all the experiments. When not specified otherwise, mice were fed a standard diet containing $280 \mathrm{mg} / \mathrm{kg}$ of carbonyl iron.

Blood was collected by tail vein puncture for complete blood count (CBC) at 3, 5, 8 and 9 months of age. Mice were sacrificed when they were 3 or 9 months old and blood was collected for determination of the transferrin saturation. Liver, spleen and kidneys were dissected, weighed and snap-frozen for RNA and protein analysis or dried for iron quantification or processed for FACS analysis. BM cells were harvested and processed for methylcellulose assay, flow cytometry or RNA analysis. Duodenum was washed and formalin-fixed for Perls staining.

BM transplantation was performed as described by Nai et al. ${ }^{13}$ and in the Online Supplementary Material. The CBC was evaluated monthly. At sacrifice animals were analyzed as above.

A subset of Ncoa4-ko mice was crossed to C57BL/6N Hbbt ${ }^{13 / 4}$ animals ${ }^{14}$ (Jackson Laboratories, Bar Harbor, ME, USA) obtaining $\mathrm{Ncoa4}^{+/-}$and $\mathrm{Ncoa4}^{+/} / \mathrm{Hbb}^{\text {th3/+ }}$ progenies on a mixed C57/129 back- ground; these animals were back-crossed generating Ncoa4 $/ \mathrm{Hbb}^{t h 3 /+}, \mathrm{Ncoa}^{+/} / \mathrm{Hbb}^{t h 3 /+}$ and $\mathrm{Hbb}^{t h 3^{3 /+}}$ mice. Blood was collected for $\mathrm{CBC}$ evaluation from 1, 2 and 4-month old animals of both genders.

All mice were maintained in the San Raffaele Institute animal facility in accordance with European Union guidelines. The study was approved by the Institutional Animal Care and Use Committee of San Raffaele Institute.

\section{Treatments}

For the induction of iron deficiency, Ncoa4-ko mice of both genders were fed an iron-deficient diet containing less than $3 \mathrm{mg} / \mathrm{kg}$ of carbonyl iron, (SAFE, Augy, France) for 6 months starting when they were 3 months old. Transplanted mice were fed a standard diet for 2 months and then the iron-deficient diet until sacrificed 5 months after BM transplantation.

For the evaluation of duodenal iron absorption, 9-month old wt and Ncoa4-ko mice (of both genders) were administered $100 \mu \mathrm{L}$ of a solution containing $228.5 \mathrm{mg} / \mathrm{L}$ of the stable iron isotope ${ }^{57} \mathrm{Fe}$ (Sigma-Aldrich) by oral gavage. The animals were fasted for $16 \mathrm{~h}$ before ${ }^{57} \mathrm{Fe}$ administration and $1 \mathrm{~h}$ after gavage they were anesthetized by intraperitoneal administration of Avertin (2,2,2-tribromoethanol, $250 \mathrm{mg} / \mathrm{kg}$; Sigma-Aldrich). Blood for preparation of serum was withdrawn from retro-orbital vessels and mice were subsequently perfused transcardially with phosphate-buffered saline. Duodenum and liver were recovered, washed with phosphate-buffered saline, weighed and immediately snap-frozen.

For the induction of acute erythropoietic expansion, wt and Ncoa4-ko mice (of both genders) were treated with a single injection of erythropoietin $(0.8 \mathrm{IU} / \mathrm{g})$ or saline as a control and sacrificed $15 \mathrm{~h}$ later.

At sacrifice all animals were analyzed as described above.

\section{Phenotypic characterization}

Determination of the $\mathrm{CBC}$, transferrin saturation and tissue iron content, flow cytometry analysis, colony-forming unit assay, Perls blue staining, inductively coupled plasma mass spectrometry, western-blot analysis and quantitative real-time polymerase chain reaction were performed by standard methods. Details are provided in the Online Supplementary Material.

\section{Statistics}

Data are presented as the mean \pm standard error (SE). An unpaired two-tailed Student $t$-test (for variables with a normal distribution) or Mann-Whitney test (for variables with a nonGaussian distributions) was performed using GraphPad Prism 5.0 (GraphPad). $P$ values $<0.05$ are considered statistically significant.

\section{Results}

\section{Ncoa4-ko mice on a Sv129/J background have microcytic red cells but not anemia}

Given the significant impact of mouse strain on iron metabolism, ${ }^{15}$ we investigated the phenotype of Ncoa4-ko mice on a Sv129/J background, a strain more iron rich than C57BL/6 (AN and LS, unpublished data and Levy et al. ${ }^{16}$ ). For comparison with Ncoa4-ko C57BL/6 age-matched animals, ${ }^{6}$ the CBC was periodically determined until 9 months of age when mice were sacrificed. Differently from C57BL/6 animals, red blood cell (RBC) count, hematocrit (not shown) and hemoglobin levels were similar in Sv129/J Ncoa4-ko mice and wt littermates. Only the erythrocyte indices, mean corpuscular volume (MCV) and mean corpuscular hemoglobin $(\mathrm{MCH})$, were slightly 
lower (decreases of about $4.4 \%$ and $6 \%$, respectively) in mutant mice than in controls, at all ages analyzed, as occurred in the C57BL/6 strain 6 (Figure 1A). Bone marrow (BM) and spleen erythroid differentiation (Figure 1B) was similar in wt and Ncoa4-ko mice at 3 and 9 months of age (Figure 1C and data not shown). Ncoa4-ko BM cells generated the same number of burst-forming units-erythroid (BFU-E) and colony-forming units-granulocyte/monocyte (CFU-GM) as wt BM cells in methylcellulose assays (Figure 1D). Consistent with normal hemoglobin levels, erythropoietin (Epo) expression in the kidney was comparable in wt and Ncoa4-ko animals (Online Supplementary Figure S1A).

Overall these results show that the lack of Ncoa4 in an iron-rich background causes only mild microcytosis without anemia, preserves normal maturation and does not impair the clonogenic capcity of erythroid cells, thus excluding important differentiation defects in vivo.

\section{Ncoa4-ko mice have normal iron parameters but tissue iron retention in ferritin}

At difference from C57BL/6 mice, ${ }^{6}$ 9-month old Sv129/J Ncoa4-ko mice had transferrin saturation, serum iron levels and liver, spleen and kidney non-heme iron content comparable to those in wt mice (Table 1). However, iron retention was evident in sections of duodenum from Ncoa4-ko animals (Figure 2A), as observed in C57BL/6 mice. ${ }^{6}$

Duodenal iron accumulation was not due to increased expression of the iron-regulatory hormone hepcidin (Hamp), which was instead reduced in mutant mice (Figure 2B). The inhibition of Hamp was likely mediated by downregulation of the BMP-SMAD pathway, as suggested by the low expression of the BMP-SMAD target gene, inhibitor of differentiation 1 (Id1) (Figure 2C). This occurred in the presence of normal levels of expression of the hepcidin activators Bmpl and Bmp2 and of the hepcidin inhibitor erythroferrone (Erfe) both in BM and spleen (Online Supplementary Figure S1B-E). Despite normal liver iron content, transferrin receptor 1 (Tfr1) expression, which is inversely correlated to intracellular iron, was increased in the livers of Ncoa4-ko mice (Figure 2D), in line with results obtained in NCOA4-depleted cells. ${ }^{2}$ Tfr1 expression was also increased in the kidneys of mutant mice (Online Supplementary Figure $S 1 F$ ), despite normal kidney iron content. On the other hand, liver ferritin $\mathrm{H}$ levels were increased, as expected because of the impaired ferritinophagy (Figure 2E). Collectively, these data suggest that, irrespective of normal iron concentration, Ncoa4-ko tissues sense a signal of iron deficiency, likely due to a reduced "free" iron pool secondary to ferritin iron retention.

\section{Ncoa4-ko mice are susceptible to iron deficiency}

To characterize their capacity to release iron from the stores in chronic iron deficiency, a cohort of wt and Ncoa4-ko mice was challenged with an iron-poor diet for 6 months, starting at 3 months of age. Surprisingly this diet did not significantly affect the hematologic parameters of wt mice, likely because of the high body iron content of the Sv129/J strain. Like C57BL/6 mice, Sv129/J Ncoa4-ko mice fed with a low-iron diet developed severe anemia, especially in the last month of the diet (Figure 3A). BM and spleen erythropoiesis in Ncoa4-ko mice remained substantially comparable to that in wt animals, with similar percentages of Ter $119^{+}$cells and without evi- dent maturation differences (Figure 3B). However, Ncoa4ko cells isolated from iron-deficient mice generated significantly fewer BFU-E and CFU-GM than did wt cells (Figure $3 \mathrm{C}$ ), suggesting that severe iron deficiency impairs the clonogenic capacity of early progenitors cells lacking Ncoa4.

Iron deficiency reduced the levels of circulating and tissue iron in both genotypes. After 6 months Ncoa4-ko mice had dramatically lower transferrin saturation and serum iron levels than had wt littermates, while liver, speen and kidney content (Table 1) and Perls staining on duodenal sections (Online Supplementary Figure S2A) were comparably decreased. These results suggest that in circumstances of prolonged, chronic iron deficiency, Ncoa4-ko mice likely mobilize stored iron through NCOA4-independent mechanisms. Nevertheless, liver ferritin levels remained higher in Ncoa4-ko mice than in wt ones, consistent with reduced ferritin degradation (Online Supplementary Figure S2B). Levels of liver Hamp, Id 1 and Bmp6 expression were similarly decreased in Ncoa4-ko and wt mice (Online Supplementary Figure S2C-E). Ncoa4-ko mice showed a trend towards increased liver Tfr1 expression (Online Supplementary Figure S2F) and a significant upregulation of kidney Tr1 (Online Supplementary Figure S2G), despite iron content being comparable to that of wt mice. This is consistent with a functional iron deficiency, as observed under a standard diet. As expected, kidney Epo expression was higher in anemic Ncoa4-ko mice than in wt ones (Online Supplementary Figure S2H).

Overall these results suggest that in chronic iron deficiency impaired ferritinophagy decreases iron availability for erythropoiesis in Ncoa4-ko mice, thus causing severe anemia.

Because of the relevance of iron absorption in iron deficiency and hypoxia, ${ }^{17,18}$ we wondered whether the lack of Ncoa 4 could also affect duodenal iron uptake. To investigate this point, we treated 9-month old Ncoa4-ko and wt controls with a single dose of ${ }^{57} \mathrm{Fe}$ via oral gavage (100 $\mu \mathrm{L} / \mathrm{mouse}$ of a solution containing $228 \mathrm{mg} / \mathrm{L}{ }^{57} \mathrm{Fe}$ ) and sacrificed them $1 \mathrm{~h}$ later. ${ }^{19}$ The amount of ${ }^{57} \mathrm{Fe}$ determined via inductively coupled plasma mass spectrometry in the duodenum, serum and liver (Online Supplementary Figure S3) was similar in wt and Ncoa4-ko animals, excluding that the lack of Ncoa4 significantly impairs dietary iron uptake.

\section{Ncoa4-ko bone marrow reconstitutes normal erythropoiesis in vivo}

To investigate erythropoiesis of Ncoa4-ko mice in more depth, we used BM transplantation to assess the capacity of Ncoa4-deficient hematopoietic stem cells to reconstitute erythropoiesis. BM transplantation replaces all recipient hematopoietic lineages, spleen macrophages ${ }^{20}$ and some liver macrophages ${ }^{21}$ with donor cells. Lethally irradiated Ncoa4-ko mice were transplanted with BM cells from wt littermates (and from Ncoa4-ko as controls) (Figure 4A). Wild-type mice were transplanted with BM cells from Ncoa4-ko donors (and from wt littermates as controls) (Online Supplementary Figure S4A).

Two months after the transplant, erythropoiesis was fully recovered in animals transplanted with both Ncoa4ko and wt BM, with comparable RBC counts and hemoglobin levels (Figure $4 \mathrm{~B}$ and Online Supplementary Figure $S 4 B-E)$. This result disputes the impaired differentiation capacity of Ncoa4-ko erythroid progenitors/precursors in vivo. 
A
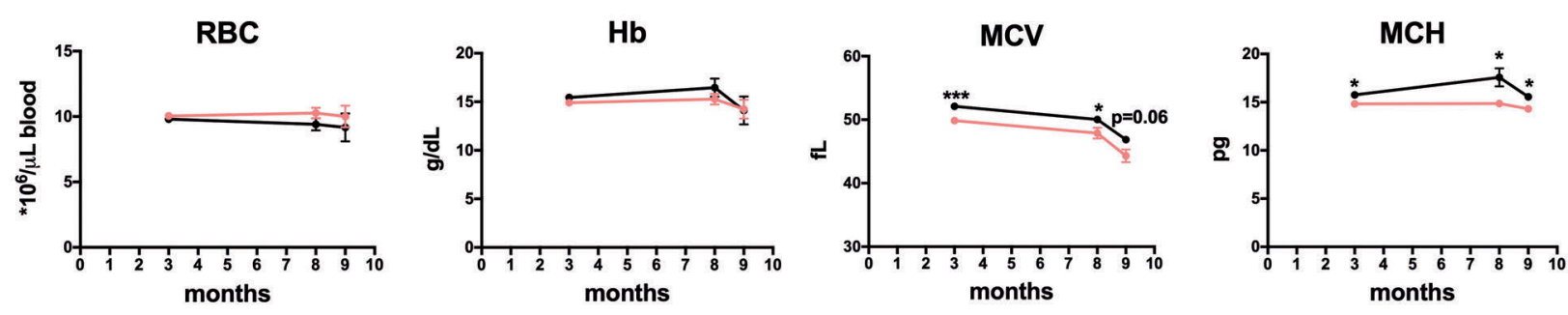

WT $\rightarrow$ Ncoa4-ko

B

\section{Bone Marrow}
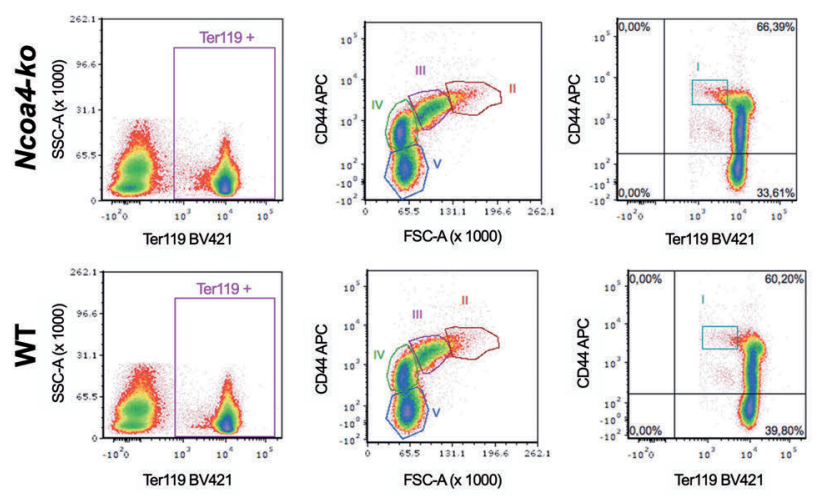
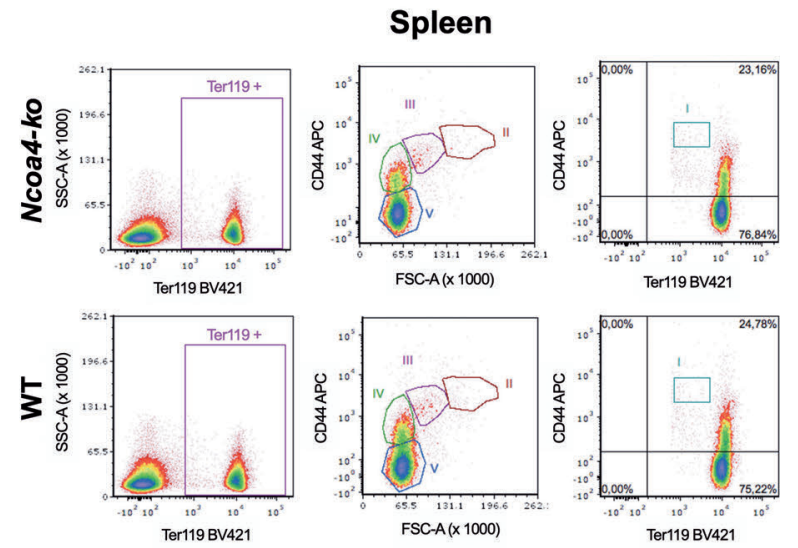

C
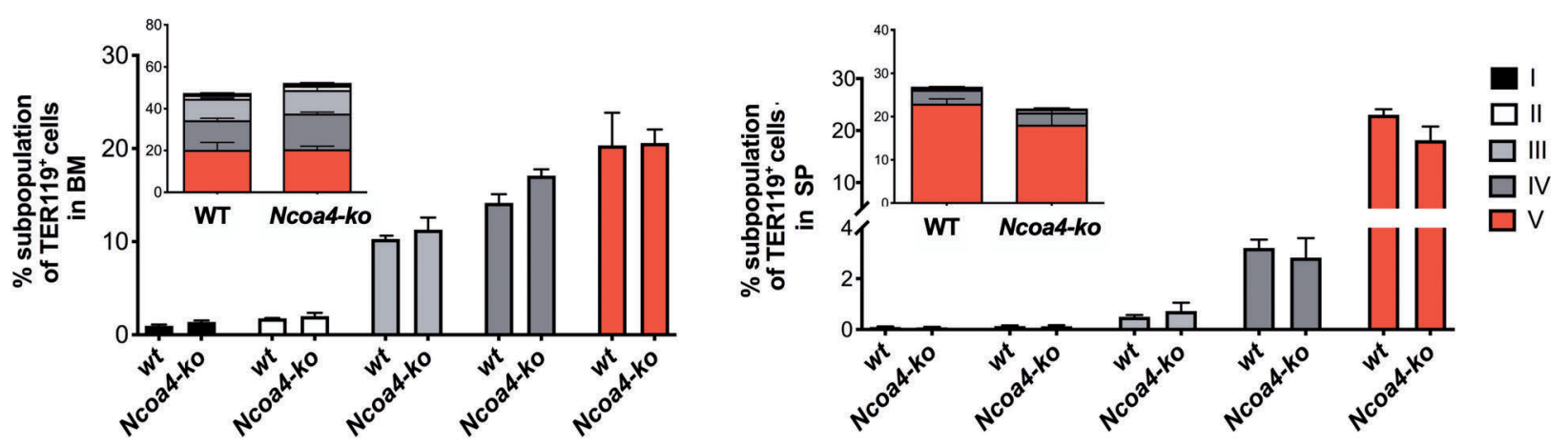

D
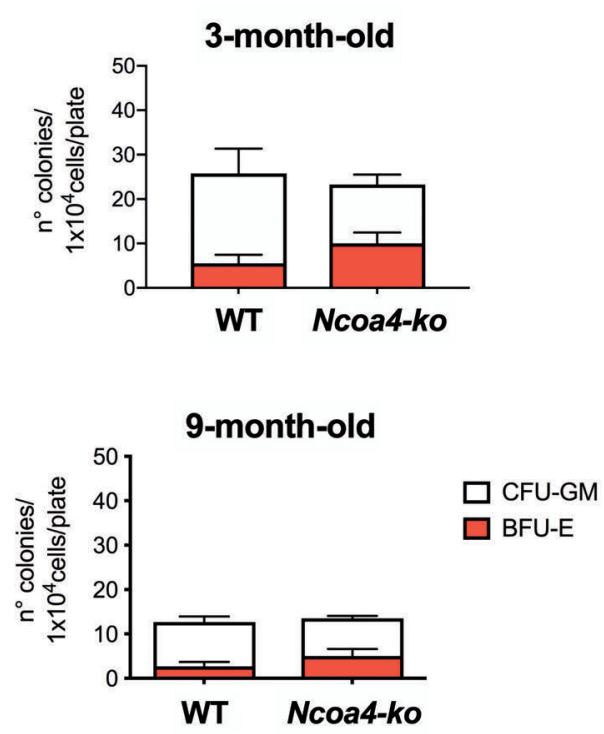

Figure 1. Analysis of hematologic parameters and erythropoiesis of wild-type and Ncoa4-ko mice fed a standard diet. Ncoa4-ko and wild-type (wt) mice (of both genders) on a Sv129/J background were fed a standard diet until sacrifice at the age of 9 months. Complete blood counts were periodically determined. (A) Values are shown for red blood cell count, hemoglobin levels, mean corpuscular volume and mean corpuscular hemoglobin. (B) Gating strategy for the analysis of erythropoiesis. Viable cells (impermeable to propidium iodide) from bone marrow and spleen were analyzed for Ter119/CD44 expression. Ter119+ cells were gated and further analyzed with respect to forward scatter and CD44 surface expression for subpopulation composition. Five clusters were identified: proerythroblasts (I), basophilic erythroblasts (II), polychromatic erythroblasts (III), orthochromatic erythroblasts and immature reticulocytes (IV), and mature red cells (V). ${ }^{13}$ The complete statistical analysis is reported in Online Supplementary Table S3. (C) Percentage of Ter119+ cells on alive cells and subpopulation composition in the bone marrow and in the spleen of 3-month old wt and Ncoa4-ko mice. The complete statistical analysis is reported in Online Supplementary Table S4. (D) Colony numbers generated from $10^{4}$ bone marrow cells of 3- and 9-month old wt and Ncoa4-ko mice. Mean values of six animals per genotype are shown. Error bars indicate the standard error. Asterisks refer to statistically significant differences between age-matched wt and Ncoa4-ko mice. $* P<0.05 ; * * * P<0.005$. RBC: red blood cells; Hb: hemoglobin; MCV: mean corpuscular volume; MCH: mean corpuscular hemoglobin; BM: bone marrow, SP: spleen; CFU-GM: colony-forming unit-granulocyte/monocyte; BFU-E: burst-forming unit-erythroid. 
Table 1. Iron parameters of 9-month old wild-type and Ncoa4-knockout mice fed a standard and an iron-poor diet.

\begin{tabular}{|c|c|c|c|c|}
\hline & \multicolumn{2}{|c|}{ Standard diet } & \multicolumn{2}{|c|}{ Iron-poor diet } \\
\hline & WT $(n=5-10)$ & Ncoa4-ko $(n=6-13)$ & WT $(n=4)$ & Ncoa4-ko $(n=5)$ \\
\hline $\mathrm{TS}(\%)$ & $57.21 \pm 2.58$ & $57.60 \pm 1.80$ & $36.70 \pm 5.03$ & $14.10 \pm 2.88 * *$ \\
\hline $\mathrm{SI}(\mu \mathrm{g} / \mathrm{dL})$ & $296.1 \pm 34.5$ & $331.9 \pm 55.6$ & $176.9 \pm 24.5$ & $74.5 \pm 13.4^{*}$ \\
\hline LIC ( $\mu$ g iron/g dry tissue) & $522.1 \pm 37.4$ & $596.6 .5 \pm 37.5$ & $136.6 \pm 10.9$ & $165.9 \pm 5.0$ \\
\hline SIC ( $\mu$ g iron/g dry tissue) & $4897 \pm 713$ & $3518 \pm 417$ & $1161 \pm 215$ & $632 \pm 155$ \\
\hline KIC ( $\mu$ g iron/g dry tissue) & $1382 \pm 168$ & $1276 \pm 122$ & $748 \pm 59$ & $772 \pm 53$ \\
\hline
\end{tabular}

TS: transferrin daturation; SI: serum iron; LIC: liver iron content; SIC: spleen iron content; KIC: kidney iron content. The values reported are the mean \pm standard error for each group. The number of animals analyzed in each group is indicated in the header. Asterisks refer to statistically significant differences between Ncoa4-knockout (ko) and wildtype (wt) mice fed the same diet. ${ }^{*} P<0.05 ;{ }^{* *} P<0.01$.

A

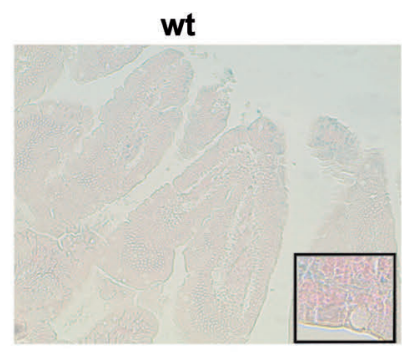

C

\section{Liver Id1}

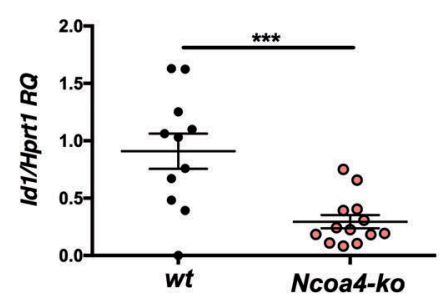

Ncoa4-ko

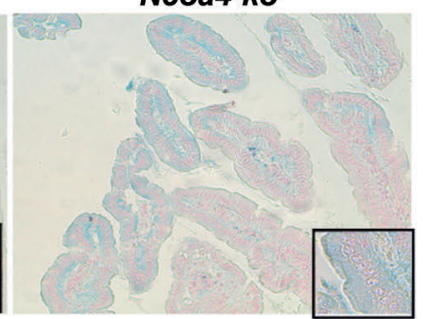

D

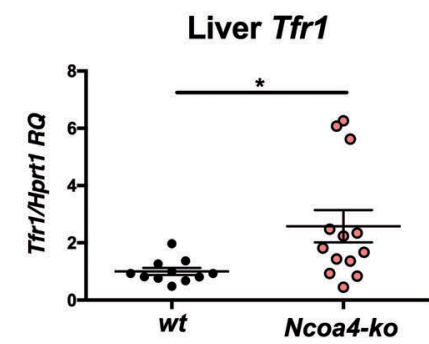

B

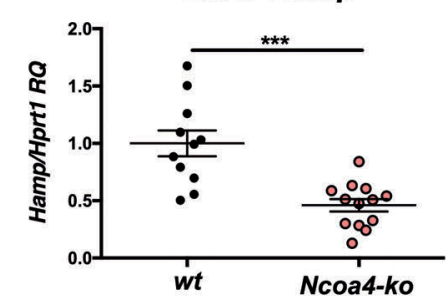

$\mathbf{E}$

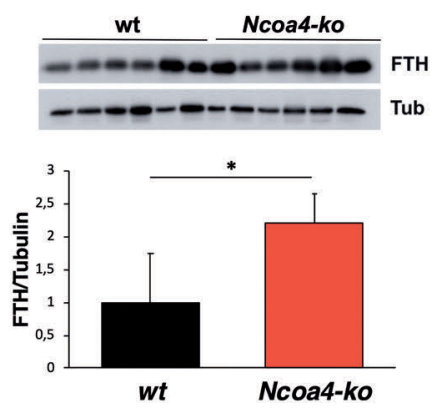

Figure 2. Analysis of the iron phenotype of 9-month old wild-type and Ncoa4-ko mice fed a standard diet. Ncoa4-ko and wild-type (wt) mice (of both genders) on a Sv129/J background were fed a standard diet until sacrifice at the age of 9 months. (A) Representative pictures of Perls staining performed on duodenal sections (thickness of section $5 \mu \mathrm{m}$; magnification 20X, 40X in the inset). (B-D) Quantitative real-time polymerase chain reaction analysis of hepcidin (Hamp) (B), inhibitor of differentiation 1 (IdI) (C) and transferrin receptor 1 (Tfr1) (D) to measure mRNA levels in the liver relative to those of hypoxanthine phosphoribosyltransferase 1 (Hprt1). Data were normalized on a wild-type mean value of 1 . (E) Western blot and relative densitometric analysis of ferritin $\mathrm{H}$ protein levels in the liver. Tubulin was used as a loading control; Mean values of 6-13 animals per genotype are shown. Error bars indicate the standard error. Asterisks refer to statistically significant differences between age-matched wt and Ncoa4-ko mice. ${ }^{*} P<0.05$; ${ }^{*} P<0.01$; $* * * P<0.005$. RQ: relative quantification; FTH: ferritin H; Tub: tubulin.

The microcytosis of Ncoa4-ko mice transplanted with wt BM was fully corrected with their MCV reaching levels of untransplanted wt mice (Figure 4B). Conversely, wt mice transplanted with Ncoa4-ko BM developed a mild microcytosis, comparable to that of germ-line Ncoa4-ko animals (Online Supplementary Figure $S 4 B-E$ ).

These experiments clearly demonstrate that the microcytosis of Ncoa4-ko mice is due to the loss of NCOA4 exclusively in BM-derived cells, either erythroid cells or macrophages or both. However, considering not only the normal erythropoiesis documented in Ncoa4-ko mice but also the ability of ko BM cells to completely reconstitute erythropoiesis in lethally irradiated animals, our conclusion is that a major defect in erythroid precursors lacking Ncoa4 is unlikely.

\section{Ncoa4-ko macrophages display impaired ferritinophagy in vivo}

To better characterize ferritinophagy impairment in Ncoa4-ko macrophages, Ncoa4-ko mice reconstituted with

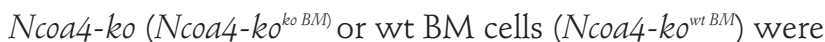
fed a low iron diet for 3 months starting 2 months after BM transplantation. The duration of this iron-deficient diet was shorter than the one used in germ-line animals to avoid the activation of the NCOA4-independent compensatory mechanisms of iron release observed in total Ncoa4-ko mice. The iron-deficient diet reduced hemoglobin levels, MCV and MCH without affecting RBC count in both genotypes, with a trend toward a more severe effect in Ncoa4-koko ${ }^{k M}$ transplanted mice (Figure 4B), confirming that the susceptibility to iron-deficiency of Ncoa4-ko mice is due to a defect in BM-derived cells. The comparable BM and spleen erythroid differentiation in the two genotypes (Figure 4C) argues against an intrinsic defect of Ncoa4-ko erythroid cells, rather suggesting that microcytosis and increased susceptibility to iron deficiency result from defective iron release by Ncoa4-ko macrophages.

In support of this interpretation, after 3 months of diet, the spleen iron content in Ncoa4-ko ${ }^{k 0} B M$ mice was higher than that in controls (Figure 4D), consistent with a defect 
A

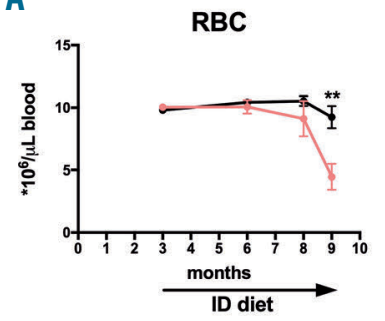

B

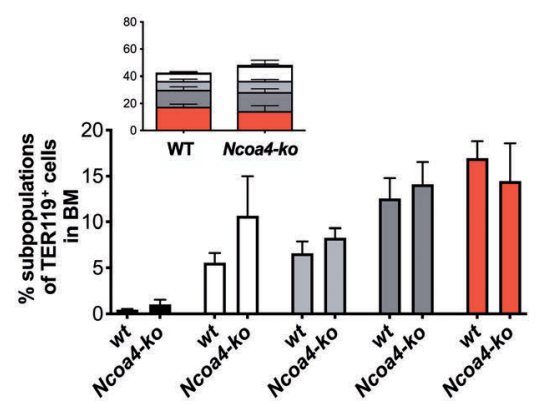

$\mathrm{Hb}$

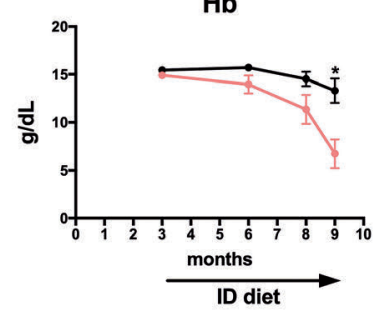

$\rightarrow$ WT

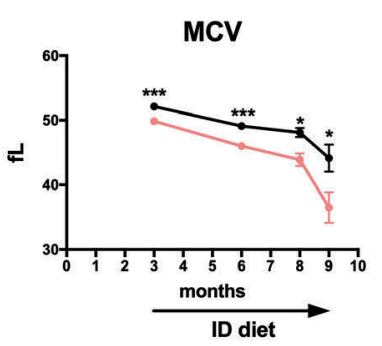

$\rightarrow$ Ncoa4-ko

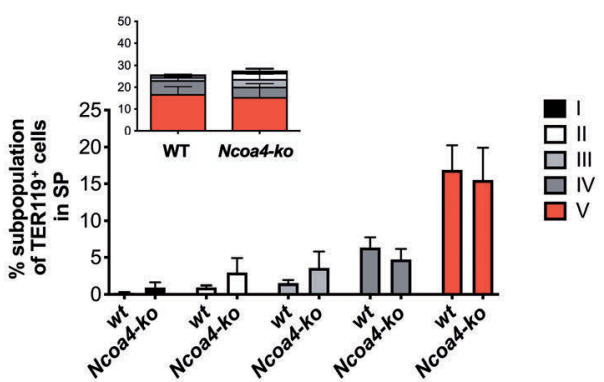

C

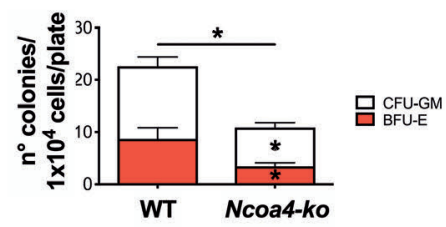

Figure 3. Hematologic parameters and analysis of erythropoiesis in wild-type and Ncoa4-ko mice fed an iron-deficient diet. Ncoa4-ko and wild-type (wt) mice (of both genders) on a Sv129/J background were fed an iron-deficient diet for 6 months starting from the age of 3 months. Complete blood counts were determined periodically. (A) Values are shown for red blood cell count, hemoglobin levels, mean corpuscular volume and mean corpuscular hemoglobin. (B) Percentage of Ter119+ cells on alive cells and subpopulation composition (determined as described in Figure 1B) both in the bone marrow and in the spleen of 9-month old irondeficient wt and Ncoa4-ko mice. The complete statistical analysis is reported in Online Supplementary Table S5. (D) Colony numbers generated from $10^{4}$ bone marrow cells of 9-month old iron-deficient wt and Ncoa4-ko mice. Mean values of four or five animals per genotype are shown. Error bars indicate the standard error. Asterisks refer to statistically significant differences between age-matched wt and Ncoa4-ko mice. $* P<0.05 ; * * P<0.01 ; * * * P<0.005$. ID: iron-deficient; RBC: red blood cells; Hb: hemoglobin; MCV: mean corpuscular volume; MCH: mean corpuscular hemoglobin; BM: bone marrow, SP: spleen; CFU-GM: colony-forming unit-granulocyte/monocyte; BFU-E: burst-forming unit-erythroid.

of Ncoa4-ko macrophages in releasing iron in vivo, despite comparable transferrin saturation (Online Supplementary Figure S5A), serum iron levels (Online Supplementary Figure $S 5 B)$ and liver iron content (Figure 4E) in the two genotypes. Splenic iron accumulation did not result from increased hepcidin levels (Online Supplementary Figure S5C), but from impaired degradation of ferritin. Indeed ferritin levels in the spleen of Ncoa4-ko $o^{k 0} B M$ animals remained higher than in mice transplanted with wt cells (Figure 4F).

Despite comparable iron content, liver ferritin levels were higher in the Ncoa4-ko $0^{k_{0} B M}$ mice than in Ncoa4-ko $0^{\text {vit BM }}$ controls (Figure 4G), suggesting impaired ferritin degradation also in this tissue. Since macrophages are the only hepatic cells derived, at least in part, from donor BM, ${ }^{21}$ these results further confirm the reduced ability of Ncoa4ko macrophages to degrade ferritin. Limited macrophage ferritinophagy, which restricts iron recycling, explains the severity of anemia in iron deficiency and supports a prevalent non-autonomous role of NCOA4 in erythropoiesis.

\section{The circulating iron levels in Ncoa4-ko mice fail to rise upon acute erythropoietic expansion}

Iron recycling is essential not only to compensate for chronic anemia but also in response to the acute expansion of erythropoiesis, such as after bleeding or stimulation with erythropoietin. To verify the role played by NCOA4 in response to an acute increase of iron demand in vivo, we exploited a published protocol, ${ }^{22,23}$ treating wt and Ncoa4-ko mice with a single injection of erythropoietin (8 IU/g body weight) to induce erythropoietic expansion, increased erythroferrone release ${ }^{24}$ and inhibi- tion of hepcidin. In normal mice, iron uptake from the diet and release from stores are enhanced to supply erythropoietic needs, resulting in a transient increase in the levels of serum iron $15 \mathrm{~h}$ after the administration of erythropoietin ${ }^{22}$ (Figure 5A). At this time point no significant changes of BM erythropoiesis were observed, while the percentage of early erythroid precursors was increased in the spleen in both wt and Ncoa4-ko mice (Figure 5B and Online Supplementary Figure S6A). The induction of splenic Erfe was comparable in both genotypes (Figure 5C) and hepcidin inhibition was even stronger in mutant mice than in wt ones (Figure 5D). Despite low hepcidin levels and differently from the situation in wt mice, transferrin saturation and serum iron levels were not increased in erythropoietin-treated Ncoa4-ko mice (Figure 5E, F). These findings indicate that the latter mice fail to mobilize iron stores in response to an acute increase of iron demand. The hypoferremia observed in the latter animals likely contributes to decrease hepcidin, suppressing the BMP-SMAD pathway, as suggested by the concomitant reduction of Id 1 mRNA, in the absence of changes in Bmp6 and Bmp2 (Online Supplementary Figure S6B-D).

\section{Ncoa4 deletion does not further worsen anemia in thalassemic mice}

To further prove the non-autonomous role of NCOA4 in erythropoiesis, we investigated the effect of Ncoa4 heterozygous or homozygous deletion in $\mathrm{Hbb}^{\mathrm{thz} / \mathrm{t}}$ animals, a model of transfusion-independent thalassemia, characterized by deficiency of $\beta$-globin chains, ineffective erythropoiesis and anemia. ${ }^{14}$ We reasoned that Ncoa4 genetic 
A

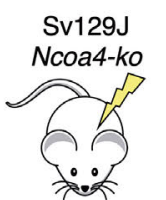

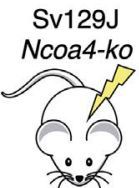

Sv129J

wild-type BM cells
B
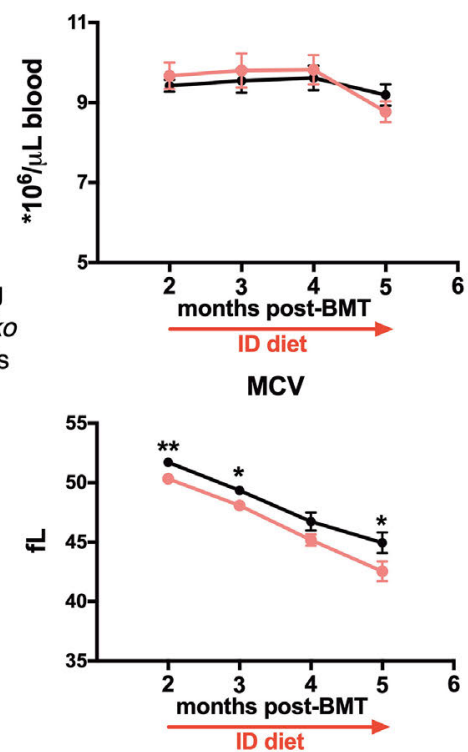

Ncoa4-kowt BM
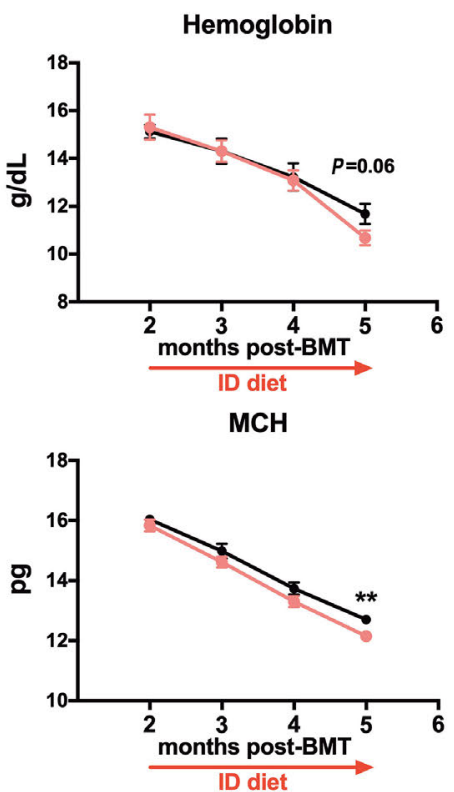

Ncoa4-koko BM

C

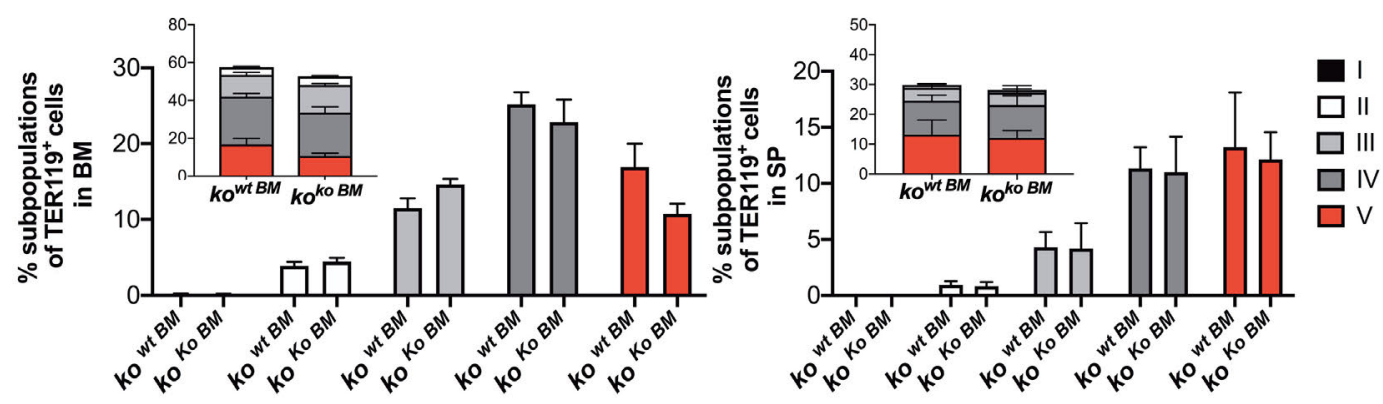

D

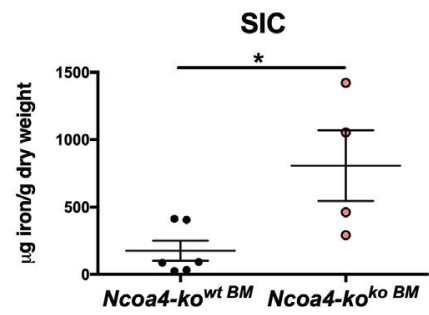

$\mathbf{F}$
E

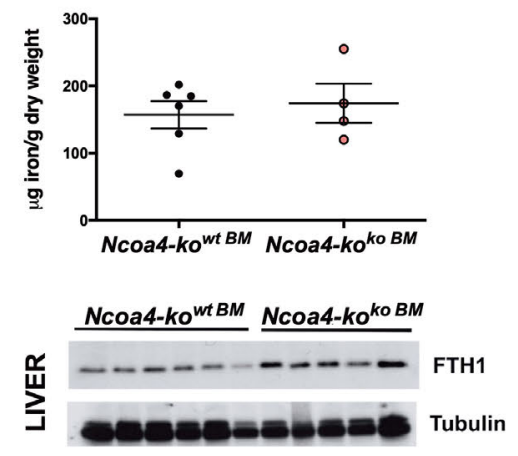

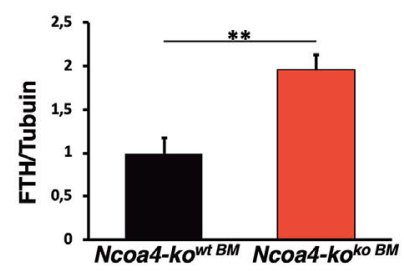

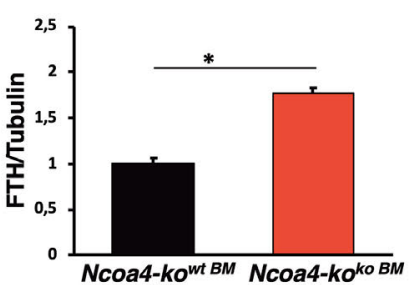

Figure 4. Hematologic parameters, erythropoiesis and iron phenotype of Ncoa4-ko ${ }^{\text {w } B M}$ and Ncoa4-ko ${ }^{\text {km }}$ mice fed an iron-poor diet. Ncoa4-ko mice on a Sv129/J back-

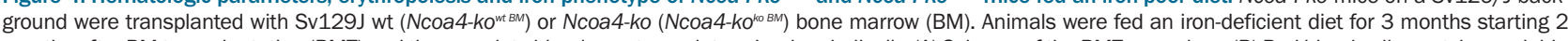
months after BM transplantation (BMT) and the complete blood count was determined periodically. (A) Scheme of the BMT procedure. (B) Red blood cell count, hemoglobin levels, mean corpuscular volume and mean corpuscular hemoglobin. (C) Percentage of Ter119+ cells on alive cells and subpopulation composition (determined as described in Figure 1B) both in the bone marrow and in the spleen of Ncoa4-ko ${ }^{\text {tBM }}$ and Ncoa4-ko ${ }^{\text {koBM }}$ mice 5 months after BMT. The complete statistical analysis is reported in Online Supplementary Table S6. (D) Spleen iron content. (E) Liver iron content. (F, G) Ferritin H protein levels in the spleen (F) and liver (G) (representative western blot, upper panels and densitometric analysis, lower panels; tubulin used as a loading control) in Ncoa4-kowtBM and Ncoa4-ko ${ }^{\text {ko BM }}$ mice 5 months after BMT. Mean values of four or five per genotype are shown. Error bars indicate the standard error. Asterisks refer to statistically significant differences between age-matched Ncoa4-kow ${ }^{\text {wt } B M}$ and Ncoa4$k^{k \circ \mathrm{BM}}$ mice. ${ }^{*} P<0.05 ; * * P<0.01$. RBC: red blood cells; MCV: mean corpuscular volume; MCH: mean corpuscular hemoglobin; SP: spleen; FTH: ferritin H. 
inactivation would worsen the erythroid phenotype of $\mathrm{Hbb}^{\mathrm{th} / \mathrm{t}}$ animals if NCOA4 was playing an important role in hemoglobinization of erythroid precursors, as suggested. ${ }^{5}$ To investigate this, we bred Ncoa4-ko mice with $\mathrm{Hb}^{\mathrm{th} 3 /+}$ animals and evaluated the CBC at 1, 2 and 4 months of age in double mutants. Ncoa 4 haploinsufficiency had no effect on anemia of $\mathrm{Hb}^{\text {th3/+ }}$ mice at any time point (Figure 6). Deletion of both Ncoa4 alleles induced a modest, statistically significant increase of RBC count at 2 and 4 month of age (Figure 6A), which may result from the iron-restricted phenotype caused by reduced iron recycling, as suggested by further decreased MCV (Figure 6C) and $\mathrm{MCH}$ (Figure 6D) of double-mutant mice compared to $\mathrm{Hbb}^{\text {th } 3 /+}$ controls.

In conclusion Ncoa4 deletion did not aggravate anemia in this model, suggesting a minor, if any, effect of NCOA4

A

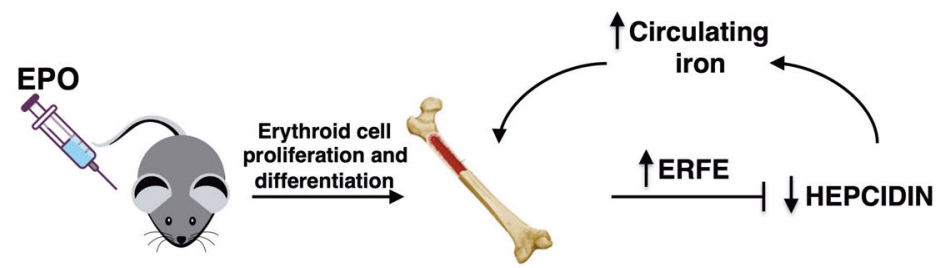

B
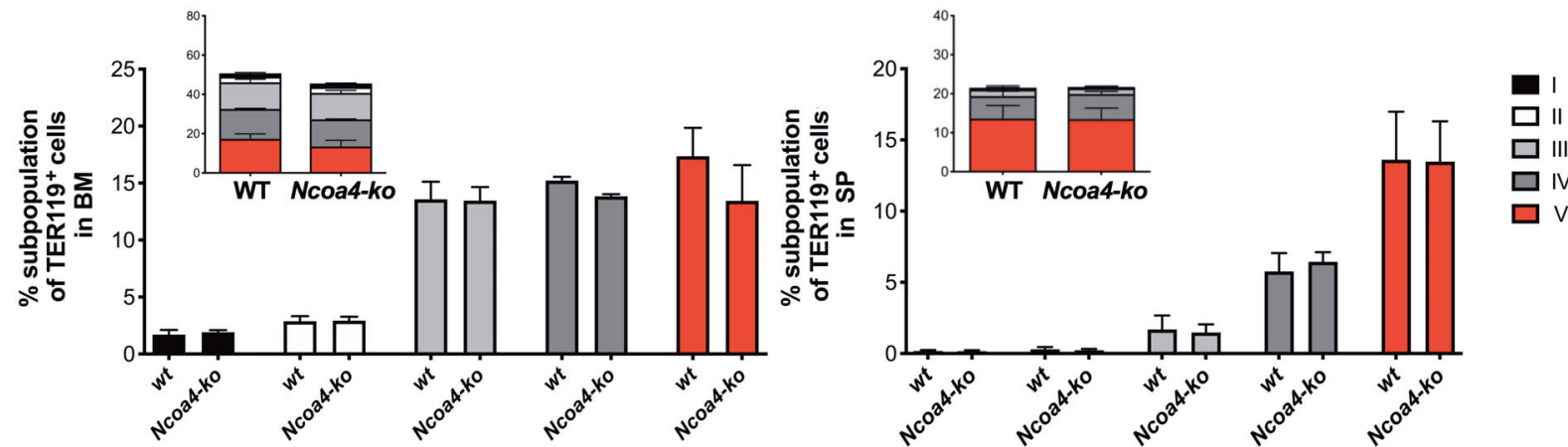

C

Spleen Erfe

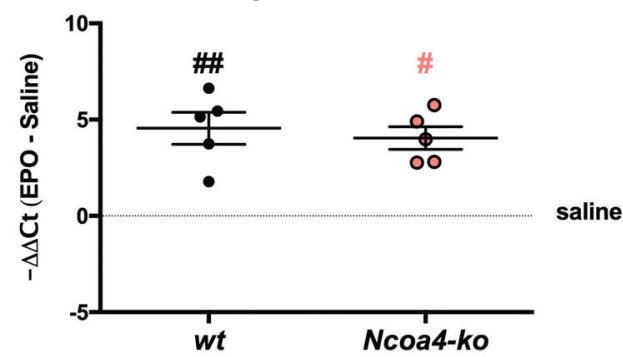

E

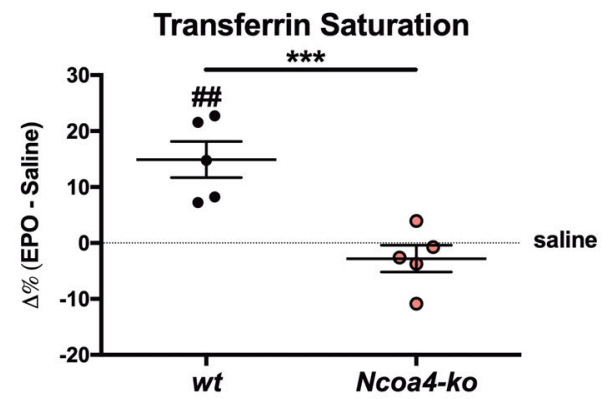

D

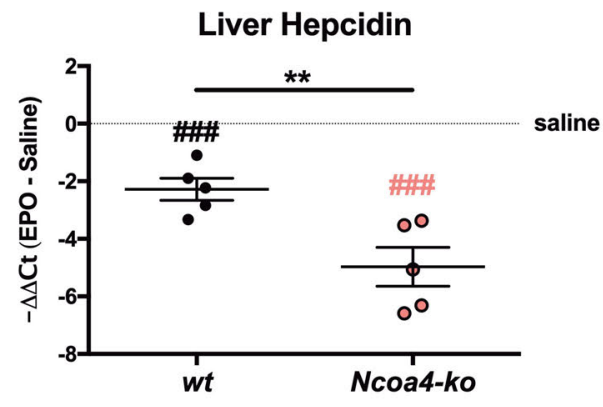

$\mathbf{F}$

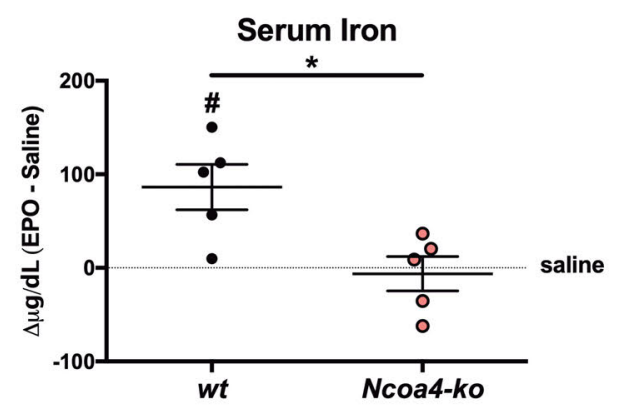

Figure 5. Erythropoiesis and iron phenotype of wild-type and Ncoa4-ko mice after an acute erythropoietin challenge. Three-month old Ncoa4-ko and wild-type (wt) mice on a Sv129/J background were treated with a single intraperitoneal injection of erythropoietin (EPO; 8 IU/g) or saline as a control and sacrificed $15 \mathrm{~h}$ later. (A) A scheme of the expected alterations of iron homeostasis induced by the EPO injection. (B) The percentage of Ter119+ alive cells and subpopulation composition (determined as described in Figure 1B) both in the bone marrow and in the spleen of EPO-treated wt and Ncoa4-ko mice. The complete statistical analysis is reported in Online Supplementary Table S7. (C) Real-time polymerase chain reaction (PCR) of splenic erythroferrone (Erfe) to measure mRNA levels relative to those of glyceraldehyde 3-phosphate dehydrogenase (Gapdh). (D) Real-time PCR of hepatic hepcidin (Hamp) to measure mRNA levels relative to those of hypoxanthine phosphoribosyltransferase 1 (Hprt1). (E) Transferrin saturation. (F) Serum Iron levels. Data in (C-F) are expressed as the difference ( $\Delta$ ) between mice of the same genotype treated with EPO or saline (dotted gray line). Mean values of five animals per genotype are shown. Error bars indicate the standard error. Asterisks refer to statistically significant differences between age-matched EPO-treated wt and Ncoa4-ko mice. $* P<0.05$; $* * P<0.01$; $* * * P<0.005$. Hashtags refer to statistically significant differences between EPO- and saline-treated mice of the same genotype. ${ }^{\# P} P 0.05$; ${ }^{\# *} P<0.01$; ${ }^{\# \#} P<0.005$. ERFE: erythroferrone; BM: bone marrow; SP: spleen. 
in erythropoiesis. The stable or even slightly improved phenotype secondary to the loss of Ncoa4 is in line with mild iron restriction, a condition described to ameliorate the thalassemic phenotype. ${ }^{25-27}$

\section{Discussion}

NCOA4-mediated ferritinophagy is essential for maintaining cell and systemic iron homeostasis. ${ }^{2.69}$ Importantly, loss of NCOA4 function in vivo induces anemia, especially in conditions of low iron. Which cell type(s) depend most on NCOA4 and ferritinophagy in order to sustain irondependent processes, and especially erythropoiesis, remains uncertain.

Here, by combining different experimental approaches, we dissected the tissue-specific role of NCOA4 and ferritinophagy in supporting RBC production and showed that the major function of NCOA4 is in iron recycling macrophages.

First, we demonstrated the relevance of total body iron as a modifier of the phenotype of Ncoa4-ko mice. Anemia is not a feature of Ncoa4-ko mice on Sv129/J, a strain with higher body iron than the C57BL/6 one (Levy et al. ${ }^{16}$ and personal data). Terminal erythropoiesis proceeds normally in Ncoa4-ko mice and BM cells isolated from both 3and 9-month old mice fed a standard diet generate the same number of BFU-E and CFU-GM as do wt cells. However, anemia is induced more easily in Ncoa4-ko mice than in controls by a prolonged iron-deficient diet. Lack of anemia in iron-replete conditions and increased susceptibility to anemia in iron deficiency suggest that
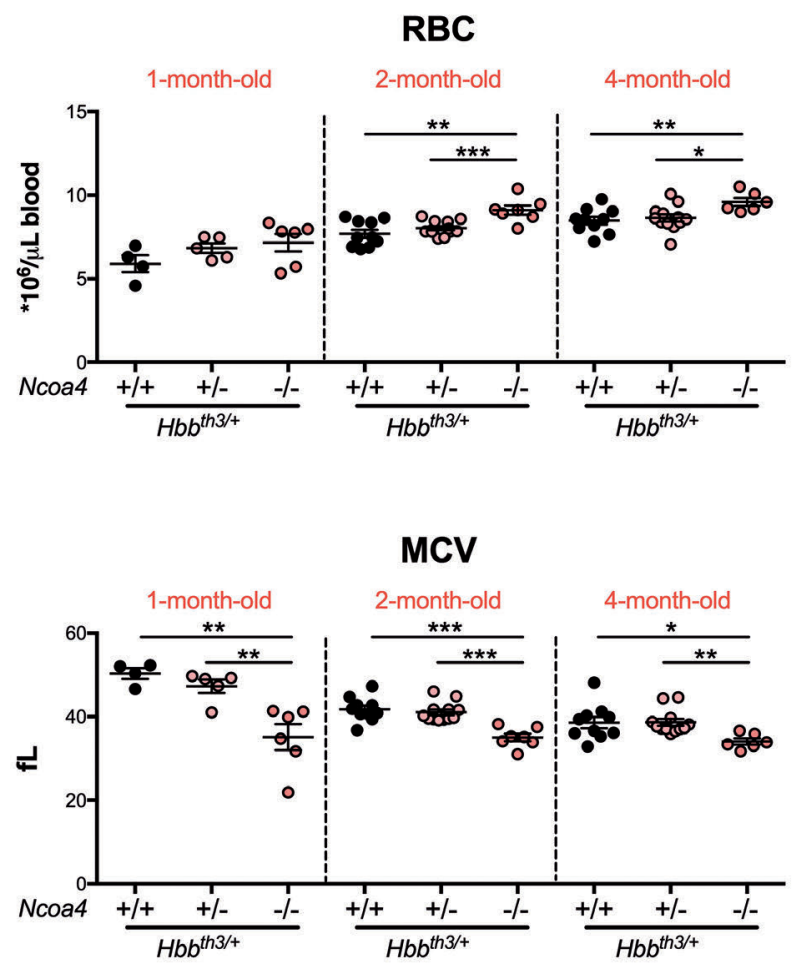

the erythroid phenotype in Ncoa4-ko mice is strongly dependent on the efficacy of iron supply. Indeed, if NCOA4 is crucial for erythroid cell differentiation and hemoglobinization, anemia would develop in Ncoa4-ko animals irrespectively of their genetic background. Intriguingly, while NCOA4 is fundamental for providing iron in acute conditions, a prolonged (6 months) irondeficient diet leads to substantial splenic and hepatic iron mobilization in Ncoa4-ko mice, suggesting that NCOA4independent mechanisms are likely activated in chronic conditions, although these mechanisms are insufficient to prevent the development of anemia.

Second, after BM transplantations Ncoa4-ko BM cells reconstituted normal RBC counts and hemoglobin levels in both wt and Ncoa4-ko recipients, with microcytosis being the only RBC abnormality. The ability to reconstitute normal erythropoiesis and normal hemoglobin levels and RBC counts proves that loss of Ncoa4 in vivo has a limited, if any, effect on the erythroid lineage. In addition, haploinsufficiency and total Ncoa4 ablation did not worsen anemia of a $\beta$-thalassemia mouse in the long term. If NCOA4 has an autonomous erythroid role, it would be expected that its deletion in a context of a constitutionally abnormal erythropoiesis would further decrease hemoglobin levels. Our results, instead, suggest that the ironrestricted phenotype of Ncoa4-ko mice protects erythroid precursors from oxidative stress and improves RBC, as observed with other iron restrictive approaches, ${ }^{25-27}$ although enhancing microcytosis.

The autonomous erythroid function proposed for NCOA4 in a recent publication was based on data from tamoxifen-inducible total Ncoa4-ko mice, which develop
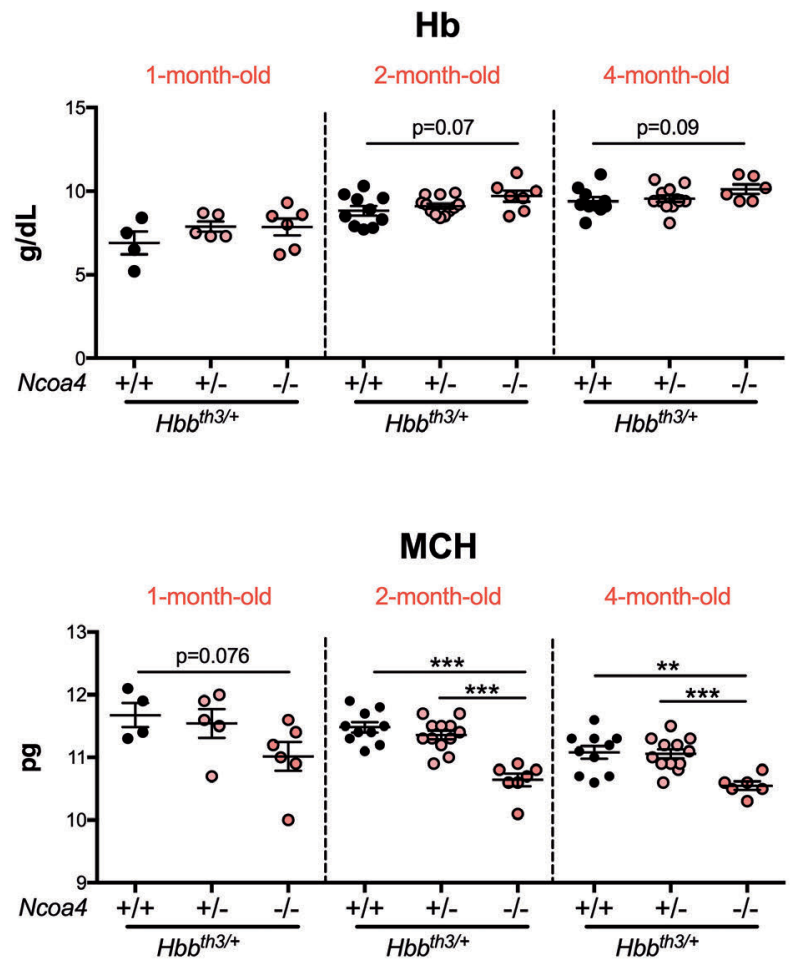

Figure 6. Hematologic parameters of $\mathrm{Hb}^{\text {th } 3 /+}$ mice with germ-line deletion of $\mathrm{Ncoa} 4$. Hematologic parameters of both male and female $\mathrm{Hbb}^{\text {th } 3 /+}$ mice with wt $\mathrm{Ncoa} 4$ $\left(\mathrm{Ncoa}^{+/+}\right)$or germ-line ablation of a single $\left(\mathrm{Ncoa}^{+} /\right)$or both $\left(\mathrm{Ncoa}_{4} /\right)$ alleles were determined at 1,2 and 4 months of age. The figure shows the values for red blood cell count, hemoglobin concentration, mean corpuscular volume and mean corpuscular hemoglobin. Asterisks refer to statistically significant differences. $* * P<0.01$; $* * * P<0.005$. RBC: red blood cell count; Hb: hemoglobin concentration; MCV: mean corpuscular volume; MCH: mean corpuscular hemoglobin. 
acute anemia after tamoxifen treatment. ${ }^{10}$ However, our recent finding that tamoxifen has a toxic effect on $\mathrm{RBC}$ production, an effect that is even stronger in mice lacking Ncoa4, weakens the authors' conclusion. ${ }^{11}$ The same authors also generated an erythropoietin receptor (Epor)Cre Ncoa4-floxed mice in which Ncoa4 genetic ablation was induced by Cre recombinase expressed under the control of the Epor promoter. ${ }^{10}$ The microcytic anemia of these animals was considered a proof of NCOA4 cellautonomous function in the erythroid compartment. However, Epor is not exclusively expressed in the erythroid lineage but also in other cell types, including macrophages. ${ }^{12}$ This raises concerns about the erythroid specificity of the model and makes the reported findings not conclusive regarding a NCOA4 self-autonomous erythroid role.

It is important to note that the modest microcytosis of Ncoa4-ko animals was reproduced by reconstituting lethally irradiated wt mice with Ncoa4-ko BM cells, while it was completely rescued by transplanting Ncoa4-ko animals with wt BM-derived cells. These findings indicate that the microcytosis resulted from the lack of NCOA4 in BMderived cells, excluding the role of other cells such as iron absorptive enterocytes, which are knocked-out in Ncoa4ko mice transplanted with wt donors. Concordantly, Ncoa4-ko mice show normal uptake and distribution of an orally administered iron isotope, ${ }^{57} \mathrm{Fe}$. Although the technique is likely unable to detect subtle differences in absorption, we concluded that iron sequestration into ferritin caused by Ncoa4 deficiency, despite inducing a condition of functional iron deficiency, does not substantially alter the HIF- $2 \alpha$-mediated orchestration of duodenal iron homeostasis ${ }^{17,18}$ in vivo, as recently shown in an in vitro cellular model in which Ncoa4 was silenced..$^{28}$

While limiting the role of NCOA4 in erythroid precursors and enterocytes, our data point to a crucial function for ferritinophagy in macrophages. Ncoa4-ko macrophages have impaired iron recycling capacity in vivo, likely secondary to their reduced ability to degrade ferritin, a defect exacerbated by acute increases of iron demand. However, this does not translate into decreased saturation of transferrin, except in conditions of acute needs, such as after the administration of erythropoietin. Our observation is in line with results obtained in mice with selective inactivation of the iron exporter ferroportin which develop a more severe anemia than wt controls when fed an iron-poor $\operatorname{diet}^{29}$ because of their impaired iron export capacity. We also speculate that impaired ferritinophagy in the central nurse macrophages of erythroblastic islands might affect the iron supply to maturing erythroblasts, ${ }^{30}$ resulting in slightly microcytic RBC when circulating iron is normal and in anemia when transferrin-bound iron is limited in iron deficiency.

NCOA4 is a multifunctional protein. We observed that $\mathrm{BM}$ cells isolated from iron-deficient Ncoa4-ko mice generate a lower number of BFU-E and CFU-GM-derived colonies compared to the numbers generated by wt animals, indicating a role for NCOA4 in early BM progenitors. We hypothesize that in mild iron deficiency, wt progenitors increase their rate of proliferation and DNA replication to sustain erythropoiesis and that this process likely requires $\mathrm{NCOA} 4$ in order to promote ferritinophagy and simultaneously control DNA replication origin activation to avoid replication stress, as suggested by Bellelli et al. ${ }^{31}$ and Federico et al. (manuscript in preparation). In agreement, a defect in clonogenic capacity was found in cells isolated from neonatal Ncoa4-ko mice, ${ }^{9}$ a condition characterized by high iron demands and functional iron deficiency. The clonogenic defect recovers in adulthood, when iron availability increases.

All together our results demonstrate the crucial role of NCOA4 in regulating iron homeostasis, in particular in response to increased iron requirements. Although a minor erythroid effect cannot be definitely excluded by all the available approaches, NCOA4 function in macrophages is the main driver of the hematologic alterations observed in Ncoa4-ko mice.

\section{Disclosures}

CC is an advisor for Vifor Iron Core and has received honoraria from Vifor Pharma. The other authors declare that they have no financial conflicts of interests.

\section{Contributions}

AN designed and performed experiments, analyzed data and wrote the manuscript; MRL, GFed, MP, VO, FCarr. and SGC performed research and contributed to the data analysis; GFer contributed to data analysis and manuscript writing; CC contributed to the experimental design and manuscript preparation; $L S$ and FCarl conceived the experiments and critically reviewed the paper. All authors approved the final version of the manuscript.

\section{Funding}

This research was supported by an EHA Josè Carreras NonClinical Junior Research Fellowship to AN, and AIRC grant IG 20793 and POR Campania FESR 2014-2020 "SATIN" grant to FCarl.

\section{References}

1. Yeh S, Chang C. Cloning and characterization of a specific coactivator, ARA70, for the androgen receptor in human prostate cells. Proc Natl Acad Sci U S A. 1996;93(11):5517-5521.

2. Mancias JD, Wang X, Gygi SP, Harper JW, Kimmelman AC. Quantitative proteomics identifies NCOA4 as the cargo receptor mediating ferritinophagy. Nature. 2014;509 (7498):105-109.

3. Dowdle WE, Nyfeler B, Nagel J, et al. Selective VPS34 inhibitor blocks autophagy and uncovers a role for NCOA4 in ferritin degradation and iron homeostasis in vivo. Nat Cell Biol. 2014;16(11):1069-1079.

4. Mancias JD, Pontano Vaites L, Nissim S, et al. Ferritinophagy via NCOA4 is required for erythropoiesis and is regulated by iron dependent HERC2-mediated proteolysis. Elife. 2015:4

5. Ryu MS, Duck KA, Philpott CC. Ferritin iron regulators, PCBP1 and $\mathrm{NCOA} 4$, respond to cellular iron status in developing red cells. Blood Cells Mol Dis. 2018;69:75-81.

6. Bellelli R, Federico G, Matte A, et al. NCOA4 deficiency impairs systemic iron homeostasis. Cell Rep. 2016;14(3):411-421.

7. An X, Schulz VP, Li J, et al. Global transcriptome analyses of human and murine termi- nal erythroid differentiation. Blood 2014;123(22):3466-3477.

8. Ryu MS, Zhang D, Protchenko O Shakoury-Elizeh M, Philpott CC. PCBP1 and NCOA4 regulate erythroid iron storage and heme biosynthesis. J Clin Invest. 2017;127(5):1786-1797.

9. Gao X, Lee HY, Li W, et al. Thyroid hormone receptor beta and NCOA4 regulate terminal erythrocyte differentiation. Proc Natl Acad Sci U S A. 2017;114(38):1010710112.

10. Santana-Codina N, Gableske S, Quiles Del Rey $\mathrm{M}$, et al. NCOA4 maintains murine erythropoiesis via cell autonomous and non-autonomous mechanisms. 
